\title{
LA DESIGUAL PARTICIPACIÓN DE LAS MUJERES MEXICANAS EN EL ACCESO Y EN LOS BENEFICIOS DE LA SEGURIDAD SOCIAL*
}

\author{
MEXICAN WOMEN'S UNEQUAL PARTICIPATION \\ ON THE SOCIAL SECURITY ACCESS AND BENEFITS
}

\author{
LA PARTICIPATION INÉGALE DES FEMMES MEXICAINES \\ À L’ACCÈS ET AUX AVANTAGES DE LA SÉCURITÉ SOCIALE
}

\author{
Berenice Patricia RAMíREZ LÓPEZ* \\ Isalia NAVA BOLAÑOS ${ }^{* * *}$ \\ Abraham GRANADOS MARTÍNEZ**** \\ Gabriel BADILLO GONZÁLEZ ${ }^{* * * *}$
}

\begin{abstract}
RESUMEN: Este artículo destaca la desigual participación de las mujeres mexicanas en el acceso y en el disfrute de los beneficios que el Estado mexicano ha propiciado como parte de la seguridad social. Se analiza la
\end{abstract}

\footnotetext{
* Recibido el 28 de febrero de 2019 y aceptado para su publicación el 6 de septiembre de 2019.

** Investigadora en el Instituto de Investigaciones Económicas de la UNAM, profesora y tutora de los programas de posgrado en Estudios Latinoamericanos y en Economía de la UNAM. Licenciada en sociología, maestra en estudios latinoamericanos, con estudios de doctorado en ciencias sociales de la UNAM.

*** Investigadora en el Instituto de Investigaciones Económicas de la UNAM. Licenciada en economía. Egresada de la maestría en demografía y el doctorado en estudios de población, de El Colegio de México. Miembro del Sistema Nacional de Investigadores nivel I.

**** Economista por la Universidad Autónoma Metropolitana, con maestría en estudios urbanos por El Colegio Mexicano y doctor en economía por la UNAM. Investigador en el Instituto de Investigaciones Económicas de la UNAM.

***** Economista por la UNAM, maestrando en demografía social en el Posgrado de Ciencias Políticas y Sociales de la UNAM. Técnico académico en el Instituto de Investigaciones Económicas de la UNAM.
} 
desigualdad desde la perspectiva de género, destacando las modalidades de inserción en el empleo y en todo tipo de ocupaciones para mostrar las diferencias salariales, la cobertura de servicios de seguridad social, particularmente el acceso a pensiones y salud; así como las desigualdades que se derivan de los apoyos que obtienen para enfrentar los riesgos del ciclo vital. El análisis se deriva de la sistematización de la información de la Encuesta Nacional de Empleo y Seguridad Social (INEGI, 2017)

Palabras clave: seguridad social, desigualdad, división sexual del trabajo, género.

ABSTRACT: This article highlights Mexican women unequal participation in access and enjoyment of the benefits provided by the Mexican state as part social security. The analysis looks at inequality from the gender perspective and it underlines the modalities of insertions in the job market, all types of occupations that show salaries differences, coverage of social security services, and, particularly, access to pensions and health. It also includes the inequalities derived from income and the support they can get to confront the risks of the life cycle. The analysis is based on the systematization of the National Survey on Employment and Social Security (INEGI, 2017).

Keywords: Social Security, Inequality, Sexual Division of labor, Gender

RÉSUMÉ: Cet article mettre l'accent sur la participation inégale des femmes mexicaine dans l'accès et la satisfaction des bénéfices fourni par l'Etat mexicain comme une partie de la sécurité sociale. On analyse l'inégalité du point de vue de la théorie des égalités des sexes en soulignant les modalités d'insertion dans le marché du travail et tout type d'occupation que montre les différences du salaire et, en particulier, avec l'accès aux pensionnes, la santé et tous les inégalités dérivées de la revenue et les appuis qu'elle peut obtenir pour confronter les risques du cycle de la vie. On fait l'analyse sur la systématisation de l'Enquête National du Travail and la Sécurité Social (INEGI, 2017).

Mots-clés: Sécurité Sociale, Inégalité, Division sexuel du travail et Genre.

SUMARIO: I. Introducción. II ¿̇Por qué la desigualdad se ubica en el centro de la reflexión? III. Desigualdad en la participación laboral de las mujeres. IV. Vulne- 
rabilidad y desigualdad en seguridad social: desventajas de las mujeres en el acceso a servicios de salud. V. Conclusiones y recomendaciones. VI. Referencias.

\section{INTRODUCCIÓN}

]

os contrastes más evidentes que muestra la desigualdad de nuestro país pueden resumirse en las siguientes comparaciones: por tamaño del Producto Interno Bruto (PIB) es la economía número 16 de 189 países, pero por índices de desigualdad se ubica en el lugar $142 .{ }^{1}$ De acuerdo con la Comisión Económica para América Latina y el Caribe (CEPAL) en 2017, el índice de Gini fue 0.50 , cifra que se ubica por encima del valor promedio en América Latina $(0.47){ }^{2}$

Por su parte, el Consejo Nacional de Evaluación de la Política de Desarrollo Social ${ }^{3}$ muestra que el decil X concentra 35.4\% del ingreso nacional, mientras que al decil I le corresponde solamente 1.9\%. Además, registra al 43.4\% de la población en condición de pobreza, si nos referimos a las mujeres, el porcentaje de pobreza sube a $44 \%$, pero es destacable el incremento a $79 \%$ para el caso de las mujeres indígenas. En las personas mayores, el nivel de pobreza general que se registra es de $41 \%$. En este contexto, la seguridad social en México ha dado por resultado un sistema excluyente y desigual que exacerba esta condición con las reformas llevadas a cabo en 1995 en el Instituto Mexicano del Seguro Social (IMSS) y en 2007 en el Instituto de Seguridad y Servicios Sociales de los Trabajadores del Estado (ISSSTE).

La información estadística disponible y sistematizada por este grupo de investigación, destaca que 57.2\% de la población ocupada se encuentra en condiciones de informalidad, por lo tanto, no está inscrita a la seguridad social y 71.4\% de las personas de 65 años y más de edad, no cuentan con una pensión de retiro, por jubilación o vejez.

1 FMI, "Perspectivas de la economía mundial", Washington, DC, octubre de 2017, p. 105, disponible en: https://mmm.imf.org/es/Publications/WEO/Issues/2017/09/19/world-econo mic-outlook-october-2017; PNUD (2017) Informe sobre Desarrollo Humano México 2016, p. 116, disponible en: https:// wmw.undp.org/content/dam/mexico/docs/Publicaciones/PublicacionesReduccionPobreza/InformesDesarrolloHumano/idhmovilidadsocial2016/PNUD\%20IDH2016.pdf.

2 Cepal, Panorama Social de América Latina 2018, Santiago de Chile, CEPAL, 2019, p. 229. disponible en: https:// repositorio.cepal.org/bitstream/ handle/11362/44395/11/S1900051_es.pdf.

3 Coneval, "Informe de Evaluación de la política de desarrollo social, 2018", México, CONEVAL, 2019, p. 254, disponible en: https://mmw.coneval.org.mx/Evaluacion/IEPSM/IEPSM/ Documents/IEPDS_2018.pdf. 
Desde la perspectiva de la desigualdad de género, este artículo revisa el acceso y las prestaciones de las mujeres en los sistemas de seguridad social en México, se sustenta revisando su participación en el ámbito laboral, en los niveles salariales, en el acceso a pensiones y servicios de salud, con la finalidad de concluir si este enfoque es suficiente para la comprensión del fenómeno o qué otros indicadores y consideraciones deberían de ser incorporados, para que pueda apoyar de mejor forma la construcción de la política pública.

Para ello se desarrollan cinco apartados. El primero, incluye una revisión de los paradigmas que han ubicado a la desigualdad en la relación Estado, mercado y sociedad. El segundo, explora las desigualdades en la participación laboral de la población femenina, particular atención se dedica a la revisión de la situación de las mujeres que laboran en la informalidad y las implicaciones de quedar excluidas de la seguridad social. El tercer apartado aborda la participación de las mujeres en el trabajo doméstico y de cuidados no remunerado y su vínculo con la ausencia de protección social. Enseguida, se revisa la desprotección en las edades avanzadas. El último apartado se centra en revisar la situación de las mujeres mayores en el acceso a los servicios de salud.

\section{II. ¿POR QUÉ LA DESIGUALDAD SE UBICA EN EL CENTRO DE LA REFLEXIÓN?}

La desigualdad social y económica como realidad del mundo contemporáneo, ha atrapado nuevamente la atención del análisis académico, de haberse considerado un proceso inevitable que acompaña al crecimiento económico, ${ }^{4}$ y que en parte suscribía el principio de crecer para distribuir, ha pasado a situarse desde diversas corrientes del pensamiento económico y de perspectivas de teoría económica, como un obstáculo al crecimiento y al desarrollo, ${ }^{5}$ pronunciándose por la perspectiva de distribuir para crecer. Especialmente

4 Cortés, Fernando, "Desigualdad económica en México: enfoques conceptuales y tendencias empíricas”, Estudios Sociológicos, México, vol. 30, núm. extraordinario, El Colegio de México, 2012, pp. 157-189; Kuznets, Simon, "Economic Growth and Income Inequality", Economic Growth and Structure, Nueva York, 1965, pp. 257-287; Prebisch, Raúl, "Raul Prebisch: un aporte al estudio de su pensamiento”, Comercio Exterior, vol. 37, núm. 5, mayo de 1987, pp. 13-30.

5 CEPAL, Pactos para la igualdad. Hacia un futuro sostenible, Santiago de Chile, Naciones Unidas, CEPAL, 2014. Disponible en: https://repositorio.cepal.org/bitstream/handle/11362/36692/ LCG2586SES353s_es.pdf? sequence=6; CEPAL, Horizontes 2030: la igualdad en el centro del desarrollo sostenible, Santiago de Chile, Naciones Unidas, CEPAL, 2016, disponible en: https:// repositorio. cepal.org/bitstream/ handle/11362/40159/4/S1600653_es.pdf; Piketty, Thomas, El capital en el siglo 
porque con la mundialización y el poder del capital financiero, pareciera que las rentas rinden más que el trabajo y que las instituciones del Estado ya no son garantía para impulsar una igualdad social relativa.

Las causas de la desigualdad tienen una relación directa con la forma como se construyó la riqueza mundial; ya sea a través de ahorro e inversiones adecuadas, patrimonios heredados o por prácticas de despojos y expropiaciones que dieron lugar a una incesante acumulación que ha modificado el espacio económico y la vida social. Lo que interesa destacar en esta reflexión es la extrema concentración de la riqueza, así como la desigualdad de género. Oxfam documenta que $82 \%$ del crecimiento de la riqueza mundial del último año ha ido a parar a manos del 1\% más rico, mientras que a la mitad más pobre de la población mundial no le ha llegado nada de ese crecimiento, lo que demuestra que los actuales niveles de desigualdad extrema superan con mucho lo que podría justificarse por el talento, el esfuerzo y el riesgo de innovar. La mayor parte de la desigualdad extrema es debida a herencias, monopolios, o relaciones de nepotismo o de connivencia con los gobiernos. ${ }^{6}$

En un sistema de mercado como el capitalista, hay evidencia que demuestra que cuando el mercado es el único que asigna a partir de un sistema de precios, como preponderantemente lo ha venido haciendo en las últimas cuatro décadas, la concentración se polariza, y lo que más llama la atención recientemente, es que se observa en un contexto de lento crecimiento de la economía mundial. Ello ha llevado a analizar la influencia que los sistemas de dominación y la construcción o ausencia de un estado de bienestar o estado social dirigido a generar o limitar los niveles de desigualdad. ${ }^{7}$ Es decir, la desigualdad tiene que ver con las necesidades de acumulación, pero también con las características del Estado y la construcción del poder, con la legitimación

XXI, trad. de Eliane Cazenave-Tapie Isoard, México, Fondo de Cultura Económica, 2014, p. 663.

6 OXFAM, "Premiar el trabajo, no la riqueza", Gran Bretaña, Oxfam Internacional, Cowley, enero de 2018, disponible en: https:// wmw-cdn.oxfam.org/s3fs-public/file_attachments/bpreward-work-not-wealth-220118-es.pdf.

7 Offe, Claus, Contradicciones en el Estado de Bienestar, trad. Antonio Escohotado, Madrid, Alianza Editorial, 1994, vol. 647, p. 309; Esping-Andersen, Gosta, Fundamentos Sociales de las economias postindustriales, trad. Francisco Ramos, Barcelona, Ariel, 2000, p. 272; Brachet, Viviane, "Contienda y dominación: una propuesta para teorizar la desigualdad", Estudios Sociológicos, vol. 30, núm. extraordinario, El Colegio de México, 2012, pp. 111-156; Oszlak, Oscar, "Formación histórica del Estado en América Latina: elementos teórico-metodológicos para su estudio", en Oszlak, Oscar (ed.), Lecturas sobre el Estado y las políticas públicas: Retomando el debate de ayer para fortalecer el actual, San José, Costa Rica, EDUCA, 2007, p. 115. 
propia del régimen expresado en las acciones de los gobiernos, de las élites, de los grupos de interés o de presión, entre otros. Si el papel del Estado social ha sido mediar en los conflictos para evitar desigualdad extrema y aunque pareciera que no es posible superar contradicciones estructurales que tienen que ver con las sociedades de clases y la propiedad privada, una posibilidad de modificar condiciones extremas ha sido mediante políticas de inclusión, de participación, que generen oportunidades y posibilidades que permitan superar la añeja consigna que Dubet ${ }^{8}$ analiza adecuadamente, describiendo cómo el principio de que cada quien merece la posición que ocupa se ha consolidado como resultado de la modernización.

Deaton ${ }^{9}$ señala que evidentemente el capitalismo ha traído un mundo diferente para buena parte de la humanidad, les ha dado en el ámbito del bienestar la oportunidad de vivir más años, de tener menos susceptibilidad frente a las enfermedades, pero también ha ocasionado otro males, más enfermedades asociadas a la creciente urbanización y las consecuencias de la contaminación ambiental, pero también reconoce que a pesar de los logros, la sociedad es hoy más desigual que hace 300 años y que el bienestar entendido como cosas buenas para la vida, bienestar material, físico, y psicológico, con salud y felicidad, educación y capacidad de participar como ciudadano, no se ha alcanzado.

Un principio ético-normativo que busca cambio de perspectiva pone la atención en la búsqueda de la equidad, idea asociada a la justicia redistributiva, ${ }^{10}$ y que se ha llevado a una proyección mayor bajo el principio de igualdad en la perspectiva de derechos humanos, protegidos por distintos instrumentos nacionales e internacionales, que para el caso del análisis de género y de inclusión se ha acompañado del principio de la no discriminación. ${ }^{11}$

De esta forma se han determinado acciones concretas para que se puedan superar las desventajas, identificadas como factores determinantes de la inequidad y de la exclusión. Los enfoques de género toman como punto de partida la desigualdad a la que se suman las condiciones impuestas por la

8 Dubet, Francois, ¿Por qué preferimos la desigualdad?: (aunque digamos lo contrario), trad. de Horacio Pons, Buenos Aires, Siglo XXI, 2016, p. 121.

9 Deaton, Angus, El gran escape. Salud, riqueza y los origenes de la desigualdad, trad. de Ignacio Perrotini, México, Fondo de Cultura Económica, 2015, p. 403.

10 Rawls, John, Teoría de la justicia, trad. de María Dolores González, México, Fondo de Cultura Económica, 2015, 1995, p. 549.

11 Altamirano, Melina y Gómez, Flamand (coords), Desigualdades en México, México, El Colegio de México-Siglo XXI, 2018, p. 120. 
sociedad patriarcal que ha imperado en los últimos siglos y que ha dificultado avances sustanciales en la participación de las mujeres.

La seguridad social en México se caracteriza por ser de carácter laborista, concentrándose en la cobertura del trabajo asalariado, subordinado y formal, lo que origina su carencia de universalidad. La construcción institucional, los arreglos políticos y los desequilibrios del mercado de trabajo, en la que están representados el empleo y los salarios condujeron a la diversidad de sistemas de pensiones de carácter excluyente y desigual, que coadyuvan a la estratificación social por condición laboral, salarial y de género.

\section{DESIGUALDAD EN LA PARTICIPACIÓN LABORAL}

DE LAS MUJERES

Tradicionalmente, los factores asociados a la participación laboral se han vinculado con variables macroeconómicas como la oferta y la demanda de trabajo, el grado de urbanización y el desarrollo de las economías modernas o con variables individuales como la edad y la escolaridad. ${ }^{12}$ Los datos censales históricos publicados por el Instituto Nacional de Estadística y Geografía (2015), revelan que México se ha convertido en un país predominantemente urbano con una economía dinámica apoyada tanto en la industria como en los servicios y que además del cambio en la organización productiva se han presentado importantes transformaciones en la estructura de la población, caracterizados por la disminución de la mortalidad, el control de la fecundidad y la presencia de una población mayoritariamente joven con potencial productivo.

Como resultado de los cambios económicos, urbanos y demográficos, la población tiene mayor acceso a los bienes y servicios públicos, como la educación que se ha universalizado al menos en el nivel básico. Sin embargo, aún existen grandes desigualdades en el acceso a la seguridad social contributiva y sus prestaciones como la salud o las pensiones por vejez, que en gran medida se explican por las diferencias en la participación laboral por género asociadas con la menor participación de las mujeres, la inserción laboral tardía y la rotación entre el trabajo de mercado y el hogar.

Al contrastar la participación laboral de las mujeres mexicanas con los países de América Latina encontramos que México se encuentra por debajo

12 Kirsch, Henry, "La participación de la mujer en los mercados de trabajo en Latinoamérica", Notas de Población, Naciones Unidas, Comisión Económica para América Latina y el Caribe (CEPAL), 1975, pp. 19-41. 
de la media en la región. Si nos comparamos con las economías de la región como Brasil, Argentina y Uruguay se observan importantes rezagos que persisten incluso al cotejar con otros países como Costa Rica, Chile o Colombia, que en la década de 1990 iniciaron en niveles similares o inferiores a los de México (cuadro 1).

\section{CUADRO 1}

PORCENTAJE DE MUJERES QUE PARTICIPAN EN EL MERCADO LABORAL EN PAÍSES SELECCIONADOS DE AMÉRICA LATINA, 1990-2018

\begin{tabular}{|c|c|c|c|c|c|c|c|}
\hline País & 1990 & 1995 & 2000 & 2005 & 2010 & 2015 & 2018 \\
\hline Uruguay & 46 & 49 & 52 & 53 & 55 & 56 & 56 \\
\hline Argentina & 44 & 47 & 49 & 49 & 46 & 47 & 47 \\
\hline Brasil & 42 & 47 & 51 & 55 & 55 & 53 & 53 \\
\hline Venezuela & 42 & 45 & 47 & 50 & 51 & 51 & 50 \\
\hline $\begin{array}{c}\text { América } \\
\text { Latina } \\
\text { el Caribe }\end{array}$ & 40 & 43 & 47 & 50 & 51 & 51 & 52 \\
\hline México & 33 & 38 & 39 & 41 & 43 & 44 & 44 \\
\hline Costa Rica & 33 & 36 & 39 & 45 & 44 & 49 & 46 \\
\hline Chile & 32 & 34 & 36 & 38 & 47 & 50 & 51 \\
\hline Colombia & 30 & 34 & 42 & 49 & 56 & 59 & 59 \\
\hline
\end{tabular}

Fuente: elaboración propia con datos del Banco Mundial.

La mayoría de los estudios han encontrado que el trabajo de las mujeres depende de variables asociadas con los roles de género tradicionales; ${ }^{13}$ el contexto socioeconómico, la dimensión urbano-rural, la escolaridad y la edad, ${ }^{14}$ la situación conyugal, el número de hijos y la edad de los hijos, la presencia de personas dependientes en el hogar y la carga de trabajo doméstico, ${ }^{15}$ la estruc-

13 Rendón Gan, Teresa, Trabajo de hombres y trabajo de mujeres en el México del siglo XX, México, CRIM-PUEG-UNAM, 2003, p. 225.

14 Chisterson, Bruce et al., "Los múltiples condicionantes del trabajo femenino en México”, Estudios Sociológicos, vol. 7, núm. 20, mayo-agosto de 1989, pp. 251-280.

15 Cerrutti, Marcela y Zentero, René, "Cambios en el papel económico de las mujeres entre las parejas mexicanas”, Estudios Demográficos y Urbanos, vol. 15, núm. 1, 2000, pp. 65-95. 
Esta revista forma parte del acervo de la Biblioteca Jurídica Virtual del Instituto de Investigaciones Jurídicas de la UNAM http://www.juridicas.unam.mx/

tura del hogar y las horas de trabajo no remunerado, ${ }^{16}$ han sido las variables más significativas para explicar los menores niveles de participación femenina.

Como se ilustra en la gráfica 1 , la reproducción de la desigualdad laboral inicia en las etapas tempranas de formación educativa, cuando la principal actividad de los jóvenes es el estudio, a los 29 años la mayoría de los jóvenes concluye su vida académica e inicia el tránsito hacia la vida adulta con una acentuada diferenciación por sexo. Los hombres suelen tener una transición casi directa entre la escuela y el trabajo, en cambio, más de la mitad de las mujeres postergan su ingreso al mercado laboral para casarse, por el embarazo o debido a responsabilidades familiares y del hogar. El ingreso tardío tendrá efectos para el resto de la vida laboral expresados no sólo en el acceso desigual a los beneficios de la seguridad social, sino también en menores ingresos, condiciones de trabajos más precarios y mayor vulnerabilidad frente a los riesgos que se presentan a lo largo de la vida.

\section{GRÁFICA 1}

PORCENTAJE DE LA POBLACIÓN 15+ POR PARTICIPACIÓN LABORAL, POR SEXO Y TRABAJO DOMÉSTICO EN MUJERES. MÉXICO 2017

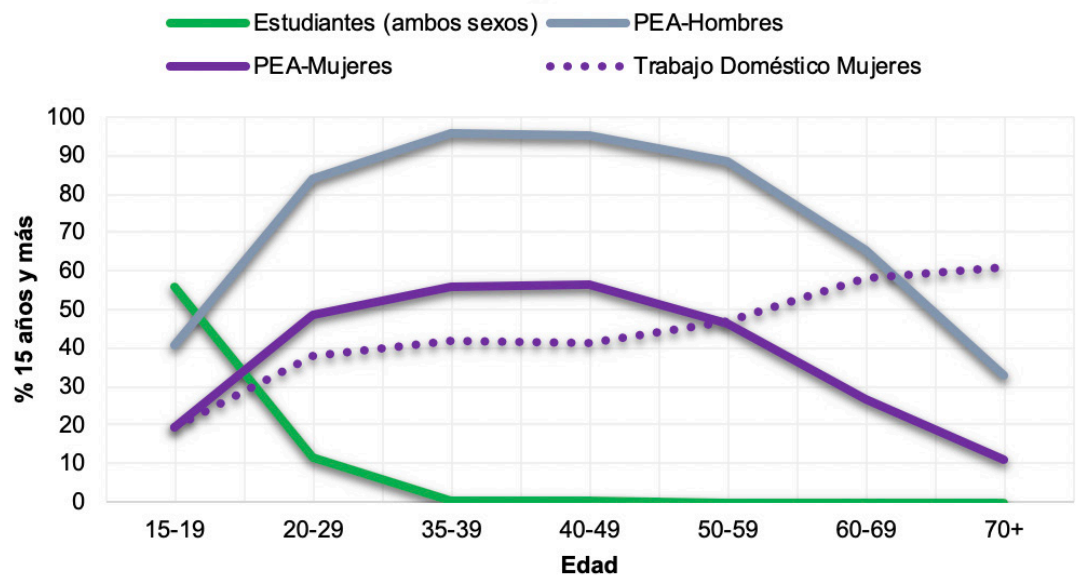

Fuente: elaboración propia con datos de INEGI, ENOE 3T-2017.

16 Sánchez, Armando et al., "Determinantes de las horas de trabajo de las mujeres en México: un enfoque de pseudopanel (2005-2010)", Revista de la CEPAL, núm. 120, diciembre de 2016, pp. 127-139. 
Investigaciones recientes de la OIT (2016) ${ }^{17}$ encontraron que la segregación ocupacional es un factor que influye en las desigualdades de los ingresos laborales, al colocar a las mujeres en trabajos donde la remuneración suele ser menor y la oferta laboral más limitada. Los datos de la ENOE muestran que las mujeres participan poco en actividades típicamente masculinas como la agricultura, la construcción o el transporte. En cambio, son más activas en el comercio y los servicios. Algunas actividades que también absorben una parte significativa del trabajo femenino como la preparación de alimentos o el trabajo doméstico remunerado son casi exclusivas de las mujeres y son al mismo tiempo actividades que reproducen los roles de género, especialmente los asociados al trabajo doméstico y de cuidados no remunerado. Aunque los roles tradicionales no son invariables y hay perspectivas de cambio entre las generaciones más jóvenes y educadas, aún se observan importantes rezagos en la segregación profesional por razones de género y en la incorporación femenina en carreras como enfermería, trabajo social o magisterio y una participación incipiente en las profesiones masculinizadas como ingeniería y ciencias básicas.

\section{Informalidad laboral}

Recordemos que el concepto de informalidad laboral se integra de dos grandes dimensiones. La primera se refiere al tipo o naturaleza de la unidad económica, es decir, a los trabajadores que realizan sus actividades en la unidad económica de los hogares. Mientras que la segunda aglutina a los trabajadores que no cuentan con el amparo institucional que otorga la seguridad social. $^{18}$

En 2017 el número de trabajadores en el empleo informal alcanzó los 29.9 millones de personas, cifra que representó $57.2 \%$ de la población ocupada. Según tipo de unidad económica empleadora, los trabajadores en el sector informal fueron 13.9 millones, 7.2 millones en el ámbito de la empresas, gobierno e instituciones, 2.3 millones en el trabajo doméstico remunerado y

17 OIT, "Las mujeres en el trabajo: tendencias de 2016", Ginebra, Organización Internacional del Trabajo, 2016, disponible en: https://www.ilo.org/womsp5/groups/public/---dgreports/--dcomm/---publ/documents/publication/woms_483214.pdf.

18 Ramírez, Berenice et al., "Las raíces de la desigualdad y de la exclusión previsional en México: propuesta para su rediseño”, en Rodríguez, Ignacio y Vommaro, Pablo (coords.), Desigualdades, exclusión y crisis de sustentabilidad en los sistemas previsionales de América Latina y el Caribe, Buenos Aires, Clacso, 2018, pp. 143- 171. 
6.9 millones en el ámbito agropecuario. Al desagregar las cifras por sexo se aprecia que $57.5 \%$ de la población ocupada femenina se encentraba en la informalidad, mientras que entre la población masculina esta cifra fue $57.0 \%$.

Uno de los factores que más contribuye a la desigualdad es la condición de informalidad que prevalece entre las mujeres ocupadas. Aunque las diferencias en los niveles de informalidad observados entre hombres y mujeres no son significativas, la desigualdad en los niveles de participación, la segregación ocupacional y la rotación entre el hogar y el mercado configuran un escenario más adverso para las mujeres, donde la informalidad juega un papel importante como soporte para quienes cuentan con poca experiencia laboral o que necesitan conciliar la vida doméstica con el trabajo de mercado.

La participación de las mujeres en condiciones de informalidad comúnmente se asocia con el incremento de la mano de obra femenina, resultado de los mayores niveles de escolaridad y la reducción de los ingresos familiares. ${ }^{19}$ Así como la facilidad de entrada en los mercados laborales, la flexibilidad en el trabajo y la compatibilidad entre el trabajo doméstico y de cuidados no remunerado y el trabajo de mercado. Para las más jóvenes los empleos desprotegidos sin seguridad y prestaciones laborales, pueden ser una puerta de entrada y la posibilidad de acumular experiencia laboral de cara a la vida adulta, mientras que, en las edades intermedias la inserción en unidades económicas del sector informal es un recurso de empleabilidad para quienes forman parte de la población no económicamente activa (PNEA) y buscan conciliar la dinámica familiar con el trabajo.

Además, las desigualdades de género en el mercado laboral también se explican a través de los procesos de segregación horizontal y vertical. La segregación horizontal lleva a que las mujeres participen en ocupaciones femeninas de acuerdo con la división sexual del trabajo. La segregación vertical explica la mayor participación de las mujeres en puestos de baja jerarquía (pisos pegajosos) y las pocas posibilidades de ocupar puestos jerárquicos (techo de cristal, glass ceiling). ${ }^{20}$

Destaca que las mujeres participan menos en trabajos formales durante su vida adulta y los periodos en los que acumulan tiempo de trabajo para acceder a las prestaciones de la seguridad social es menor en comparación con los

19 Freije, Samuel, "El Empleo Informal en América Latina y el Caribe: Causas, consecuencias y recomendaciones de política”, Banco Interamericano de Desarrollo, 2002, disponible en: bttps:/ / ideas.repec.org/p/idb/brikps/3772.html.

20 Rodríguez, Corina, "La cuestión del cuidado: ¿El eslabón perdido del análisis económico?”, Revista CEPAL, núm. 106, 2012, pp. 23-36. 
Esta revista forma parte del acervo de la Biblioteca Jurídica Virtual del Instituto de Investigaciones Jurídicas de la UNAM

hombres, quienes pueden permanecer por mayor tiempo en trabajos formales. En el caso de las mujeres mayores la participación en el sector informal se vincula más con la posibilidad de continuar participando en el mercado laboral, aunque un porcentaje importante lo hace bajo arreglos laborales de tipo familiar sin pago o ingreso regulares.

La gráfica 2 da cuenta de la heterogeneidad entre trabajadores subordinados remunerados, por tipo de ocupación es notorio el mayor porcentaje de mujeres sin prestaciones; según el sector de actividad económica, el terciario, particularmente los servicios son donde aparece la mayor desprotección femenina; en función del tipo de unidad económica, la brecha más amplia aparece en el trabajo doméstico remunerado, particularmente porque los hombres participan poco en esta actividad y, por lo tanto, tienen poca representatividad. Al incorporar la atención médica, $43.6 \%$ de las mujeres y $45.9 \%$ de los hombres no cuentan con el acceso a las instituciones de salud.

\section{GRÁFICA 2}

TRABAJADORES SUBORDINADOS REMUNERADOS Sin ACCESO A PRESTACIONES DE LEY. MÉXICO, 2017 (PORCENTAJES)*

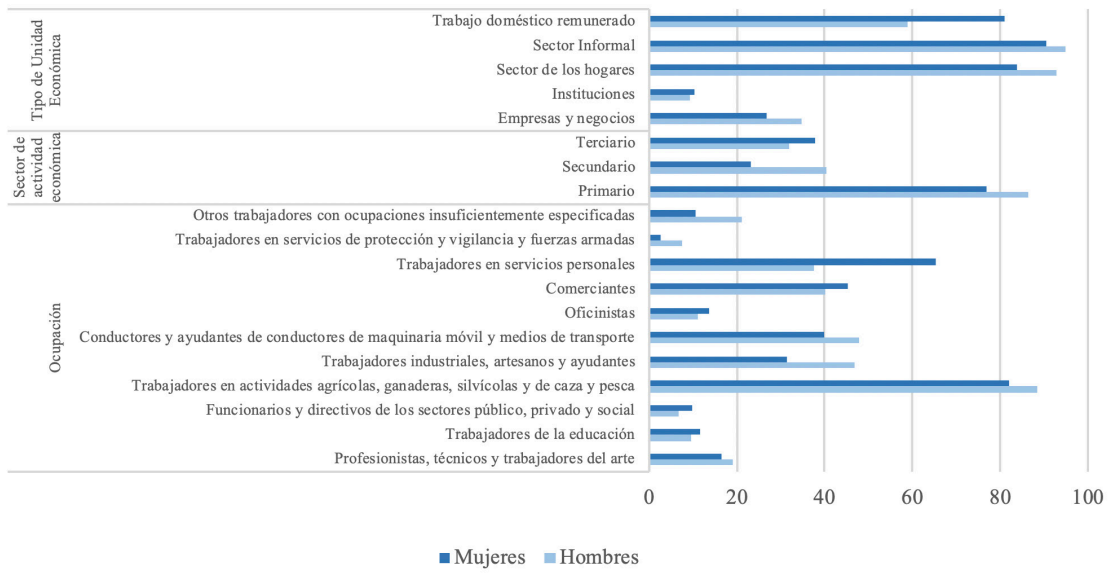

Fuente: elaboración propia con base en ENOE-INEGI, 2017-III.

Nota: proporción de trabajadores subordinados y remunerados que no tienen acceso a ninguna de las prestaciones laborales de ley (aguinaldo, vacaciones con goce de sueldo y reparto de utilidades) con respecto al total de trabajadores subordinados y remunerados. En este indicador no se considera la atención médica. 
Además, son notorias las brechas por niveles de ingresos, en el rango de ingresos más bajo "hasta un salario mínimo" aparece una mayor participación de las mujeres, $22.5 \%$ en comparación con los hombres, 12.6\%. Algo similar ocurre en el rango "más de 1 hasta 2 salarios mínimos", donde estos porcentajes son 33.5 y $28.5 \%$, respectivamente. Es notoria la mayor participación de hombres que reciben "más de 2 y hasta 3 salarios mínimos", esta tendencia se mantiene en los siguientes rangos de ingresos. En el rango más alto "más de 5 salarios mínimos" aparecen sólo $4.3 \%$ de las mujeres y $7.2 \%$ de los hombres. Además, entre quienes no reciben ingresos, es mayor la participación de las mujeres, $8.1 \%$, en comparación con $7.3 \%$ de sus congéneres varones.

Las relaciones de género conllevan asignaciones sociales y roles que condicionan la vida pública o privada de las personas, con ello, se incide en las oportunidades de crecimiento profesional y de reconocimiento, o limitaciones sociales y responsabilidad de actividades poco valoradas, como las realizadas al interior del hogar, pero que sustentan el funcionamiento de la familia, la sociedad y la economía.

Las consecuencias en las condiciones de las mujeres son diversas, ya que mantienen amplias cargas de trajo doméstico y de cuidado no remunerado a lo largo del ciclo vital y suelen tener limitado descanso aún en edades avanzadas. Con datos de la Encuesta Nacional de Empleo y Seguridad Social ${ }^{21}$ se registra que las mujeres destinan 20.8 horas promedio a la semana a realizar los quehaceres de su hogar, en tanto los hombres para la misma actividad dedican 6.9 horas promedio a la semana. Al considerar esta misma actividad, realizar los quehaceres de su hogar, para los grupos de edad de 50 a 59 años y de 60 a 69 años se presentan incrementos en el promedio de horas destinadas por parte de las mujeres, ya que utilizan 24.7 y 23.9 horas promedio, respectivamente, para el caso de los hombres de estos dos grupos de edad, se tienen 7.6 y 8.1 horas promedio a la semana, respectivamente.

\section{La desigualdad en el trabajo doméstico y de cuidados no remunerado}

En el caso mexicano, a lo largo de la construcción de los sistemas de seguridad social ha prevalecido una perspectiva laborista, que otorga prioridades a la protección de los trabajadores asalariados, urbanos y organizados, exclu-

${ }^{21}$ ENESS, tercer trimestre de 2017, INEGI, disponible en: bttp:/ / mmw. beta.inegi.org. $m x$ /pro gramas/eness/2017/ default.btml. 
yendo otras ocupaciones y trabajos. ${ }^{22}$ En específico, quedan fuera las mujeres que participan exclusivamente en la esfera privada, reservada al trabajo doméstico y de cuidados no remunerado, como consecuencia de la lógica que impone la división sexual del trabajo. ${ }^{23}$

En 2017, entre la población femenina, la PNEA fue 57.2\% y entre la población masculina 22.0\%. A su vez, dentro de la PNEA, 73.1\% de las mujeres y $9.4 \%$ de los hombres se dedicaron a los quehaceres del hogar. La edad media de estas mujeres dedicadas al hogar fue 45 años y $77.3 \%$ nunca cotizó. Se trata de un sector significativo de la población que queda excluido de la protección formal contra las contingencias económicas y sociales.

Considerar el trabajo doméstico y de cuidados no remunerado es importante porque es un trabajo que contribuye a la sostenibilidad de la vida humana y a la reproducción de la fuerza de trabajo. ${ }^{24}$ Además, se reconoce que la protección a este grupo de la población debe ser un derecho ciudadano que otorga la certeza de contar con un nivel básico de bienestar. ${ }^{25}$ Un avance en esta dirección es el modelo de afiliación a la seguridad social de las trabajadoras no remuneradas en el hogar desarrollado en Ecuador, que permite reconocer el trabajo doméstico y de cuidados, que mayoritariamente realizan las mujeres, a través de esquemas no contributivos. ${ }^{26}$

\section{La desprotección en las edades avanzadas}

La situación de exclusión y desigualdad en el mercado laboral que experimentan las mujeres a lo largo de la vida tiene consecuencias en el acceso a la seguridad social en las edades avanzadas.

22 Ramírez, Berenice et al., op. cit.

23 Molyneux, Maxine, "Más allá del debate sobre el trabajo doméstico", en Rodríguez, Dinah y Cooper, Jennifer (comp.), El debate sobre el trabajo doméstico, México, UNAM, Instituto de Investigaciones Económicas, 2005, pp. 209-250; Carraco, Cristina, "La sostenibilidad de la vida humana: ¿Un asunto de mujeres?”, Mientras tanto, núm. 82, 2001, pp. 43-70.

24 Carraco, Cristina, op. cit.; Seccombe, Wally, "El trabajo del ama de casa en el capitalismo", en Rodríguez, Dinah y Cooper, Jennifer (comp.), El debate sobre el trabajo doméstico, México, UNAM, Instituto de Investigaciones Económica, 2005, pp. 175-207.

25 Sojo, Ana, "Protección social en América Latina. La desigualdad en el banquillo", Santiago, CEPAL, 2017, p. 246.

26 Guamán, Adoración y Lorete, Raúl, "La afiliación a la seguridad social del trabajo no remunerado del hogar: el modelo de ecuador como ejemplo para un debate necesario", Revista Latinoamericana de Derecho Social, núm. 28, 2019, pp. 71-101. 
Las cifras que proporciona la ENESS 2017 dan cuenta de la poca cobertura de la seguridad social, $71.3 \%$ de las personas de 65 y más años no contaban con una pensión derivada de contribuciones. La diferencia en las pensiones de mujeres y hombres es evidente, ya que prácticamente del total de pensiones obtenidas por los hombres (99.8\%) son de forma directa, en cambio, en las mujeres es $52.9 \%$, el resto corresponde a pensiones derivadas. Es decir, se evidencia la poca participación laboral de las mujeres de forma remunerada y su acceso a las pensiones se resume a obtenerlo principalmente de forma indirecta. Lo cual, tiene incidencia en las limitadas oportunidades económicas de las mujeres que inciden en la toma de decisiones y el empoderamiento económico.

Resulta relevante cómo las mujeres de 65 y más años de edad se concentran en aquellas pensiones derivadas de la viudez y orfandad, $47.1 \%$ de las mujeres las reciben, contrario al caso de los hombres, para quienes este tipo de pensión no representa el uno por ciento del total de pensionados. Entre la población masculina sobresale la pensión por retiro, vejez y cesantía, 47.8\% de los pensionados las mencionan, en tanto que las mujeres que declaran contar con este tipo de pensión son 18.6\%, para el tipo de jubilación o tiempo de servicio es menor la disparidad por sexo, las mujeres representan $31.0 \%$ y los hombres $46.2 \%$.

$\mathrm{Al}$ recibir una pensión mujeres y hombres logran solventar riesgos de vulnerabilidad y precariedad en la vejez, sin embargo, un reto por resolver en el país es el bajo monto que reciben por estas pensiones. Es destacable que cerca de la mitad de las mujeres (47.6\%) con pensiones reciban solamente hasta un salario mínimo, lo mismo se presenta para más de una tercera parte de los hombres (36.8\%) con pensiones. Al considerar pensiones hasta de dos salarios mínimos se encuentran datos preocupantes, ya que tres de cada cuatro personas reciben pensiones mensuales de hasta dos salarios mínimos. Esta condición de bajo monto de pensiones se agudiza para el caso del IMSS, en donde más de la mitad de las mujeres y más de $40 \%$ de hombres reciben hasta un salario mínimo mensualmente. Asimismo, se registra que ocho de cada diez personas reciban hasta dos salarios mínimos de pensión mensualmente en el IMSS. Esta concentración de pensiones con montos tan bajos para importante proporción de mujeres y hombres es menos dramática para el caso del ISSSTE, ya que cuatro de cada diez personas reciben hasta dos salarios mínimos de pensión mensual. Estas condiciones limitadas de ingreso se revierten para el caso de mujeres y hombres pensionados en Pemex, la Sedena o la Marina, debido a que prácticamente $90.0 \%$ cuentan con pensiones mayores de dos salarios mínimos (cuadro 2). 


\section{CuAdro 2}

POBLACIÓN DE 65 Y MÁS AÑOS PENSIONADA SEGÚN MONTO MENSUAL. MÉXICO, 2017

\begin{tabular}{|l|c|c|c|}
\hline Institución & $\begin{array}{c}\text { Hasta 1 } \\
\text { salario mínimo }\end{array}$ & $\begin{array}{c}\text { Más de 1 a 2 } \\
\text { salarios mínimos }\end{array}$ & $\begin{array}{c}\text { Más de 2 } \\
\text { salarios mínimos }\end{array}$ \\
\hline Hombres & $36.8 \%$ & $37.5 \%$ & $25.7 \%$ \\
\hline IMSS & $42.1 \%$ & $39.9 \%$ & $18.0 \%$ \\
\hline ISSSTE & $10.6 \%$ & $31.7 \%$ & $57.7 \%$ \\
\hline $\begin{array}{l}\text { Pemex, Sedena o } \\
\text { Marina }\end{array}$ & $3.2 \%$ & $9.0 \%$ & $87.8 \%$ \\
\hline Otras & $16.6 \%$ & $17.6 \%$ & $65.8 \%$ \\
\hline Mujeres & $47.6 \%$ & $26.9 \%$ & $25.5 \%$ \\
\hline IMSS & $56.7 \%$ & $27.8 \%$ & $15.6 \%$ \\
\hline ISSSTE & $14.5 \%$ & $25.2 \%$ & $60.2 \%$ \\
\hline $\begin{array}{l}\text { Pemex, Sedena o } \\
\text { Marina }\end{array}$ & $1.8 \%$ & $7.8 \%$ & $90.4 \%$ \\
\hline Otras & $30.6 \%$ & $20.6 \%$ & $48.7 \%$ \\
\hline
\end{tabular}

Fuente: elaboración propia con datos de la ENESS-INEGI, 2017.

\section{VULNERABILIDAD Y DESIGUALDAD EN SEGURIDAD SOCIAL:} DESVENTAJAS DE LAS MUJERES EN EL ACCESO A SERVICIOS DE SALUD

Los factores sociales y normativos de género influyen en las condiciones de riesgo y vulnerabilidad para acceder a servicios sociales, ${ }^{27}$ derivado de las condiciones desiguales y de vulnerabilidad que enfrentan mujeres y hombres. Las condiciones como la raza y la etnicidad, el género y la edad influyen en los niveles de vulnerabilidad y riesgo de la población. ${ }^{28}$

27 Pizarro, Roberto, "La vulnerabilidad social y sus desafíos: una mirada desde América Latina", Estudios estadísticos y prospectivos, Santiago de Chile, CEPAL, 2001, disponible en: https:// repositorio.cepal.org/bitstream/ handle/11362/4762/S0102116_es.pdf? sequence=1 Eis Allowed =y.

28 IPCC, "Climate Change 2014: Impacts, Adaptation, and Vulnerability. Part B: Regional Aspects. Contribution of Working Group II to the Fifth Assessment Report of the Intergovernmental Panel on Climate Change", en Barros, V. R. et al. (coords.), Cambridge-Nueva York, Cambridge University Press, 2014, p. 688, disponible en: bttps:// wmw.cambridge.org/core/services/ 
Prevalecen desigualdades sociales en salud consecuencia de diferencias sistemáticas y estructurales entre y al interior de grupos sociales. Las condiciones socioeconómicas, la alimentación, el nivel educativo y el nivel de empleo influyen en el estado de salud y el acceso a los servicios médicos. La desigualdad de ingresos también se asocia con el estado de salud, por ello, se reconoce que en países más desiguales se presentan más limitaciones con la salud de una parte importante de la población, contrario a lo que se presenta en países con menor desigualdad. ${ }^{29}$

$\mathrm{El}$ poder se distribuye en la sociedad de forma inequitativa, en el mismo sentido, al interior de las familias también se ejerce el poder desigualmente, en este sentido, los problemas y riesgos sociales no son consecuencia de factores neutros, en cambio son resultado de las relaciones de poder y de la desigualdad de género. Ante ello, se pueden implementar acciones gubernamentales para solventar y contrarrestar estas desventajas que enfrentan las mujeres. ${ }^{30}$

Con relación al estado de salud, se registra que las mujeres padecen mayor número de problemas en su condición de salud que los hombres, se supone que es resultado de las normas de género, pero también sabemos que son quienes más acuden al médico y consecuentemente conocen mejor sus padecimientos con relación a los hombres (Salgado-De Snyder y Wong, 2007). Todo ello ha llevado a reconocer que su morbilidad presenta mayor número de problemas, asimismo, sus condiciones de salud son más complejas por su condición de género. Es decir, las actividades domésticas y de cuidados no remuneradas que realizan pueden llevarlas a enfrentar riesgos cotidianos que las conducirían rápidamente a la pérdida de autonomía (fracturas, artritis, etcétera). En este sentido, las actividades socialmente asignadas a las mujeres, de cuidar $\mathrm{y}$ atender de la familia suelen resultar en mayores cargas sociales y de cuidado de otros integrantes del hogar en detrimento del bienestar de las mujeres, dichas actividades se mantienen en el ciclo de vida y permanecen en edades avanzadas, con el cuidado de la pareja, nietos u otros integrantes del hogar.

Se puede considerar al envejecimiento como un cúmulo de diversas experiencias relacionadas con experiencias de vida, la edad biológica y la acumu-

aop-cambridge-core/content/view/E816A2701 AD893B49639DDDA5E44F72E/978110741538 6c1_p1133-1198_CBO.pdf/regional_context.pdf.

29 Bernales, M. et al., "Desafíos en la atención sanitaria de migrantes internacionales en Chile”, Rev Peru Med Exp Salud Pública, 2017; 34(2): 167-75. Doi:10.17843/rpmesp.2017.342.2510.

30 Holzmann Robert, Jorgensen Steen, "Manejo social del riesgo: un nuevo marco conceptual para la protección social y más allá”, Revista Facultad Nacional de Salud Pública, vol. 21, núm. 1, enero-junio de 2003. 
lación de riesgos, los cuales inciden en las condiciones de vida y en el estado de salud. Las mujeres representan la mayoría de las personas adultas mayores en prácticamente todos los países del mundo, ya que tienen mayor esperanza de vida que los hombres. Sin embargo, todavía prevalecen condiciones de desventaja en el bienestar de las mujeres en la vejez, en algunos casos se pueden asociar por inequidades en el envejecimiento de género relacionadas con las condiciones de pobreza y de vulnerabilidad. El hecho de que las mujeres vivan más tiempo no implica per se que se encuentren con mejores condiciones en su estado de salud, por el contrario, al incrementar la edad se eleva la posibilidad de tener problemas de salud y discapacidad. ${ }^{31}$

\section{Afiliación a salud de la población envejecida por sexo}

Resulta relevante que el acceso a servicios de salud no es muy distinto para mujeres y hombres en México, al considerar el total de la población. Sin embargo, en proporción más mujeres cuentan con Seguro Popular, por el contrario, más hombres no tienen afiliación a servicios de salud (cuadro 3). Con estos datos se podría considerar avances importantes en el acceso a servicios de salud en términos de igualdad de género. No obstante, es conveniente precisar que estos resultados son todavía limitados, en relación con mejorar el estado de salud de mujeres y hombres, debido a que la atención del sistema de salud tiene carencias y en especial el caso del Seguro Popular, ya que presenta diversas limitaciones estructurales, como en sus servicios y en su presupuesto.

\section{CUADRO 3}

AFILIACIÓN A SERVICIOS DE SALUd DE MUJERES Y HOMBRES, 2017

\begin{tabular}{|l|c|c|c|c|c|c|}
\hline Sexo & $\begin{array}{c}\text { Sin } \\
\text { afiliación }\end{array}$ & IMSS & ISSSTE & $\begin{array}{c}\text { Seguro } \\
\text { Popular }\end{array}$ & $\begin{array}{c}\text { Otra institu- } \\
\text { ción pública }\end{array}$ & $\begin{array}{c}\text { Servicio } \\
\text { privado }\end{array}$ \\
\hline Total & $17.2 \%$ & $36.3 \%$ & $4.9 \%$ & $37.8 \%$ & $3.1 \%$ & $0.6 \%$ \\
\hline Hombres & $19.2 \%$ & $36.7 \%$ & $4.4 \%$ & $36.1 \%$ & $3.1 \%$ & $0.6 \%$ \\
\hline Mujeres & $15.4 \%$ & $36.0 \%$ & $5.3 \%$ & $39.5 \%$ & $3.2 \%$ & $0.6 \%$ \\
\hline
\end{tabular}

Fuente: elaboración propia con datos de la ENESS-INEGI, 2017

31 Salgado-De Snyder, Nelly y Wong, Rebeca, "Género y pobreza: determinantes de la salud en la vejez”, Salud Pública de México, vol. 49, supl. 4, enero de 2007, pp. 515-22. 
$\mathrm{Al}$ respecto, resulta relevante destacar que el Seguro Popular es una solución limitada, ya que no logra atender todas las necesidades de salud de la población beneficiaria y absorbe a una importante proporción de personas que pueden estar desprotegidas ante eventos graves de salud. Así, el Seguro Popular no resuelve problemas estructurales, solamente cubre una limitada cobertura de salud, además enfrenta carencias operativas, por contar con limitados servicios médicos, con pocas unidades médicas, falta de personal, equipo y en especial de medicamentos, factores que se agudizan por los recursos insuficientes asignados para la operación del programa. ${ }^{32}$

$\mathrm{Al}$ revisar los datos para la población de 65 y más años se equiparan todavía más la proporción de mujeres y hombres con afiliación a servicios de salud, se registra que una de cada diez mujeres y hombres no cuentan con afiliación a servicios de salud, más de $40.0 \%$ de mujeres y hombres en edad avanzada tienen afiliación al IMSS y una tercera parte cuenta con Seguro Popular (cuadro 4). Lo cual podría representar una paridad en la atención de estos servicios para la población envejecida, no obstante, estos datos se pueden explicar en gran medida porque las mujeres son beneficiarias a estos servicios y no titulares.

De acuerdo con la ENESS 2017, la mayoría de la afiliación de las mujeres envejecidas es como esposas, viudas o madres beneficiarias y no por ser las titulares de los servicios, factores que se puede explicar por su historia de vida y por las normas sociales de género. A su vez, es relevante tener presente que existen diferencias en el estado de salud de mujeres y hombres, se reconoce que los hombres adultos mayores padecen más de enfermedades cardiovasculares o pulmonares, en tanto las mujeres reportan mayor incidencia de alta presión arterial y de diabetes. ${ }^{33}$

32 Laurell, Cristina, Impacto del seguro popular en el sistema de salud mexicano, Buenos Aires, Clacso, 2013, p. 165, disponible en: http:/ / paginas.facmed.unam.mx/deptos/sp/wp-content/ uploads/ 2015/11/ImpactodelSeguroPopular.pdf.

33 Salgado-De Snyder, Nelly y Wong, Rebeca, op.cit. 
CUADRO 4

AFILIACIÓN A SERVICIOS DE SALUd DE MUJERES

Y HOMBRES DE $65+, 2017$

\begin{tabular}{|l|c|c|c|c|c|c|}
\hline Sexo & $\begin{array}{c}\text { Sin } \\
\text { afiliación }\end{array}$ & IMSS & ISSSTE & $\begin{array}{c}\text { Seguro } \\
\text { Popular }\end{array}$ & $\begin{array}{c}\text { Otra } \\
\text { institución } \\
\text { pública }\end{array}$ & $\begin{array}{c}\text { Servicio } \\
\text { privado }\end{array}$ \\
\hline Hombres & $11.1 \%$ & $42.6 \%$ & $8.4 \%$ & $33.6 \%$ & $4.0 \%$ & $0.3 \%$ \\
\hline Mujeres & $10.7 \%$ & $43.0 \%$ & $9.2 \%$ & $33.1 \%$ & $3.7 \%$ & $0.3 \%$ \\
\hline
\end{tabular}

Fuente: elaboración propia con datos de la ENESS-INEGI, 2017.

En estas condiciones, donde las mujeres viven más tiempo que los hombres, pero tienen mayores asignaciones sociales no remuneradas en los hogares asociadas con los cuidados, se puede ver afectado su bienestar y descanso en edades avanzadas. Otro factor de desigualdad de género a considerar es el estado civil, ya que las mujeres tienden a tener mayor participación como viudas, como consecuencia de su mayor esperanza de vida, así suelen vivir solas o con hijos, nietos u otros familiares y en algunos casos pueden padecer algún tipo de violencia que afecte su salud y bienestar. ${ }^{34}$

$\mathrm{Al}$ revisar la afiliación a servicios de salud por entidad federativa de mujeres y hombres de 65 y más años se identifican tendencias similares, sin embargo, se pueden señalar ciertas diferencias regionales. Se registra una elevada proporción de mujeres y hombres sin servicios de salud en Michoacán y Veracruz, donde prácticamente una de cada cinco personas no cuenta con este servicio. Asimismo, sobresale el estado de Hidalgo por la alta afiliación a servicios de salud para el caso de los hombres de 65 y más años, contrario a lo que presentan el caso de las mujeres, donde una de cada diez no cuenta con ella (gráfica 3).

Estos datos de afiliación a servicios de salud por sexo de la población de 65+ demuestran un avance en la cobertura de salud en el país. Sin embargo, resultan ser logros que se deben considerar con reservas, dado que no se solventan los problemas de salud de la población ni de desigualdad de género, son servicios con distintos niveles y capacidad de atención, los cuales están asociados con la actividad en el mercado de trabajo, en donde las mujeres participan menos que los hombres durante el ciclo vital. De esta forma, se

\footnotetext{
34 Idem.
} 
Esta revista forma parte del acervo de la Biblioteca Jurídica Virtual del Instituto de Investigaciones Jurídicas de la UNAM http://www.juridicas.unam.mx/

convierte en asignación pendiente, ofrecer una atención integral en salud para mujeres y hombres, adecuadas a las demandas de cada grupo de población y de edad.

\section{GRÁFICA 3}

PROPORCIÓN DE MUJERES Y HOMBRES DE 65+, SIN AFILIACIÓN, POR ENTIDAD FEDERATIVA, 2017

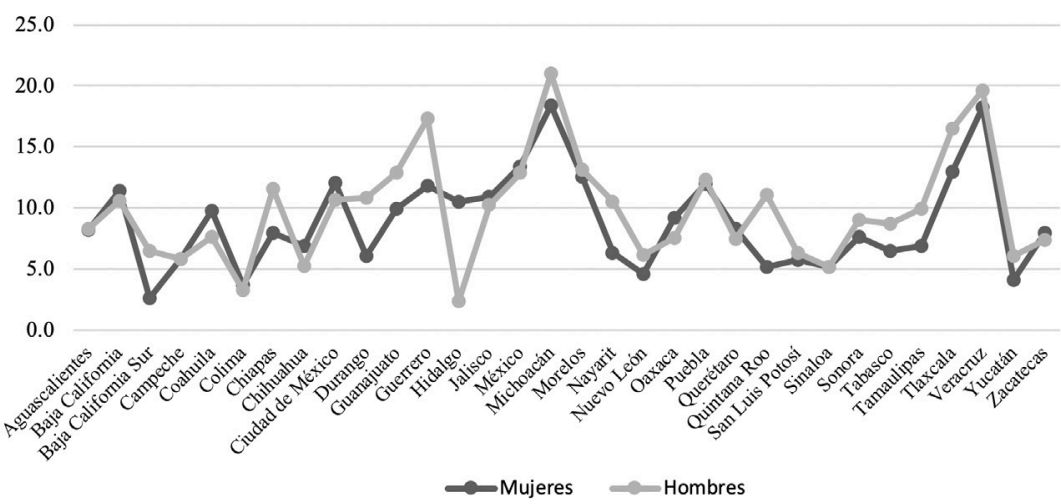

Fuente: elaboración propia con datos de la ENESS-INEGI, 2017.

Así, un objetivo a largo plazo es garantizar el acceso universal de salud, con recursos homogéneos para todas y todos, sin preferencia o detrimento del subsistema en que las personas estén afiliadas. Además, resulta pertinente para el corto y el mediano plazo focalizar acciones en favor de las necesidades más urgentes, como recortar y eliminar las brechas de género y atender grupos en pobreza. ${ }^{35}$ Asimismo, es fundamental focalizar las necesidades de salud por sexo, con estrategias específicas para enfermedades crónico-degenerativas y lesiones; apoyar actividades de cuidados a personas ancianas que se realiza en los hogares, lo cual es principalmente realizado por mujeres; ${ }^{36}$ así como promover cambios en las normas de género que deriven en actividades equitativas por sexo en el sector reproductivo de la economía, es decir, en el trabajo doméstico y de cuidado no remunerado.

35 OIT, "Protección Social de la Salud: una estrategia de la OIT para el acceso universal a la asistencia médica. Documento de consulta", Ginebra, Organización Internacional del Trabajo, agosto de 2007, disponible en: https:// www.ilo.org/public/spanish/protection/secsoc/downloads/ healthpolicy_sp.pdf.

36 Salgado-De Snyder, Nelly y Wong, Rebeca, op. cit. 
Adicionalmente, se requiere asegurar el acceso efectivo a los servicios de salud, de acuerdo con necesidades médicas y de estructura de morbilidad de la población. En este sentido, la cobertura efectiva refiere contar con disponibilidad física, financiera y geográfica de los servicios, ${ }^{37}$ para que mujeres y hombres puedan ser atendidos cuando lo requieran..$^{38}$

También, es necesario fomentar el uso de servicios de salud en edades tempranas, para garantizar mejores condiciones en la vejez, como la salud física y la mental, con énfasis en políticas preventivas que consideren las necesidades de mujeres y hombres. ${ }^{39}$ Con ello, se avanza en asegurar condiciones en el estado de salud óptimas en la vejez, con menores costos financieros y sociales, y en favor de la igualdad de género.

\section{CONCLUSIONES Y RECOMENDACIONES}

En este artículo se presenta el análisis de la desigual participación de las mujeres mexicanas en el acceso y en el disfrute de los beneficios que el Estado mexicano ha propiciado como parte de la seguridad social. Apoya al estudio la interpretación de los datos sistematizados de la Encuesta Nacional de Empleo y Seguridad Social (ENESS) realizada por el INEGI en 2017.

Mujeres y hombres viven en condiciones sociales y económicas distintas por las normas de género prevalecientes. La seguridad social ha sido excluyente y desigual por género, como consecuencia de reformas al marco normativo, por la asociación con la actividad laboral remunerada y por la estructura de género, en donde las mujeres mantienen mayor participación en actividades domésticas y de cuidados no remuneradas.

Se identifica desde la perspectiva de la desigualdad de género retos diversos por solventar en México, para mejorar las condiciones de las mujeres, en particular en la vejez, ya que la estructura demográfica evidencia una concentración futura en este grupo de edad e implicarán necesidades que todavía no se logran solventar actualmente. Se analiza la desigualdad desde la perspectiva de género, para demostrar que no es suficiente ubicar la falta o modalidades de acceso de las mujeres a las pensiones y salud, como resultado de la desigualdad social y económica del país. Sino que además ello se suma a

37 Organización Internacional del Trabajo, "Protección Social de la Salud...", cit.

38 Fajardo-Dolci, Germán et al., "Acceso efectivo a los servicios de salud: operacionalizando la cobertura universal en salud”, Salud Pública de México, vol. 57, núm. 2, 2015, pp. 180-186.

39 Salgado-De Snyder, Nelly y Wong, Rebeca, op. cit. 
los efectos de la desigualdad de género relacionada con las condicionalidades impuestas a las actividades de las mujeres asociadas fundamentalmente a la reproducción humana y al cuidado, sin considerar su aporte a la reproducción social y económica.

Como parte de los principales hallazgos, se pudo constatar que debido a que la seguridad social se fundamentó en un modelo laborista diseñado para proteger exclusivamente a los trabajadores asalariados urbanos y a los empleados públicos, excluyendo otras formas de trabajo, hasta el momento sólo se han visibilizado las condiciones de informalidad laboral, pero las actividades de cuidado y de trabajo doméstico se hacen visibles sólo como trabajadoras asalariadas subordinadas, sin que todavía se puedan incluir las actividades de trabajo doméstico y de cuidado que las mujeres realizan al interior de los hogares y que dificultan su inserción escolar y laboral.

Las mujeres participan de forma desigual en el mercado de trabajo, asociadas con los roles de género tradicionales y necesidades familiares. Además, se mantienen actividades donde predomina la participación de hombres o de mujeres, como son la agricultura, la construcción o el transporte, típicamente masculinas; contrario al caso del comercio y los servicios, donde las mujeres incrementan su actividad laboral. Adicionalmente, una importante proporción de mujeres y hombres trabajan en el sector informal y sus oportunidades de seguridad social son lejanas.

Se registra que las pensiones y los servicios de salud que reciben las personas de 65 y más años se derivan prioritariamente de su condición de beneficiarias, ya sea de su cónyuge o de sus hijos. Se destaca que la falta de titularidad ante un derecho humano acentúa la vulnerabilidad de las mujeres.

No era el espacio para profundizar sobre los efectos de la capitalización individual de administración privada que acompaña a las reformas del IMSS (1995), del ISSSTE (2007) y de otros sistemas de pensiones, pero cabe señalar que ser titular de una cuenta individual puede ser considerado como la expresión de la visibilidad de un derecho individual. Sin embargo, este modelo de financiamiento puede agudizar la desigualdad de género, debido a la mayor esperanza de vida de las mujeres, menores salarios y legislación que les solicita menor tiempo de cotización. Por lo señalado en este artículo, no se ha enfrentado con política pública, la superación de los factores que determinan la baja densidad de cotización que actualmente registran las mujeres, por las dificultades que encuentran en su inserción al empleo formal y protegido, así como las dinámicas transiciones laborales que registran en su ciclo laboral, lo 
que da por resultado que se registre mayor expulsión a la inactividad que al trabajo protegido y formal.

Así, las pensiones y los servicios de salud asistenciales van quedando cómo único apoyo de ingreso y atención en su vejez, transferencias fiscales de muy bajo monto y servicios de salud carentes de buena calidad, que no resuelven las malas condiciones de vida ni la necesaria construcción de posibilidades de bienestar para la vejez.

Es por esto que consideramos que urge la construcción de un sistema de seguridad social que resuelva contar con una base de ingreso suficiente para todas las personas en la vejez y un sistema de salud que favorezca el bienestar de la población durante el ciclo vital. La definición de la base financiera para alcanzar dicho objetivo debe considerar la transición demográfica y la perspectiva de género.

\section{REFERENCIAS}

Altamirano, Melina y Gómez, Flamand, Desigualdades en México, México, El Colegio de México-Siglo XXI, 2018.

BRACHET-MÁRQUEZ, Viviane, “Contienda y dominación: una propuesta para teorizar la desigualdad”, Estudios Sociológicos, vol. 30, núm. extraordinario, 2012.

CABIESES, Báltica et al., Vulnerabilidad social y su efecto en salud en Chile. Desde la comprensión del fenómeno hacia la implementación de soluciones, Chile, Universidad del Desarrollo, 2016, p. 118.

CARrasco, Cristina, "La sostenibilidad de la vida humana: ¿Un asunto de mujeres?”, Mientras Tanto, núm. 82, 2001, pp. 43-70.

CAstañón, Vicente y Ferreira, Olaf, "Densidades de Cotización en el Sistema de Ahorro para el Retiro en México", México, Consar, Documentos de trabajo núm. 3, 2017, disponible en: https://wmw.gob.mx/cms/uploads/ attachment/file/192977/densidad_vf.pdf.

CerrutTi, Marcela y ZENTENO, René, "Cambios en el papel económico de las mujeres entre las parejas mexicanas”, Estudios Demográficos y Urbanos, vol. 15, núm. 1, 2000.

Christenson, Bruce et al., "Los múltiples condicionantes del trabajo femenino en México”, Estudios Sociológicos, vol. 7, núm. 20, mayo-agosto de 1989. 
CEPAL, "La hora de la igualdad: brechas por cerrar, caminos por abrir. Trigésimo Tercer Período de Sesiones de la CEPAL", Santiago, CEPAL, 2010, disponible en: bttps:// repositorio.cepal.org/bitstream/ handle/11362/43405/7/ S1800082_es.pdf.

CEPAL, Pactos para la igualdad. Hacia un futuro sostenible, Santiago, Naciones Unidas, Comisión Económica para América Latina y el Caribe, CEPAL, 2014. Disponible en: https:// repositorio.cepal.org/bitstream/handle/11362/36692/L CG2586SES353s_es.pdf?sequence $=6$.

CEPAL, Horizontes 2030: la igualdad en el centro del desarrollo sostenible, Santiago, Naciones Unidas, Comisión Económica para América Latina y el Caribe (CEPAL) 2016, disponible en: https:// repositorio.cepal.org/bitstream/ handle/11362/40159/4/S1600653_es.pdf.

CEPAL, Panorama Fiscal de América Latina y el Caribe 2018: Los desafíos de las políticas públicas en el marco de la Agenda 2030, Santiago de Chile, Naciones Unidas-CEPAL, marzo de 2018, disponible en: https:/ / repositorio.cepal.org/ bitstream/ handle/11362/43405/7/S1800082_es.pdf.

CEPAL, Panorama Social de América Latina 2018, Santiago, CEPAL, 2019, disponible en: https:// repositorio.cepal.org/ bitstream/ handle/11362/4439 5/11/S 1900051_es.pdf.

Coneval, Informe de Evaluación de la política de desarrollo social, 2018, México, Coneval, 2019, disponible en: https:// www.coneval.org.mx/Evaluacion/IEPSM/ IEPSM/Documents/IEPDS_2018.pdf.

CORTÉs, Fernando, "Desigualdad económica en México: enfoques conceptuales y tendencias empíricas”, Estudios Sociológicos, México, vol. 30, núm. extraordinario, El Colegio de México, 2012.

DeATON, Angus, El gran escape: salud, riqueza y los origenes de la desigualdad, trad. de Ignacio Perrotini, México, Fondo de Cultura Económica, 2015.

DuBET, Francois, ¿Por qué preferimos la desigualdad?: (aunque digamos lo contrario), trad. de Horacio Pons, Buenos Aires, Siglo XXI, 2016.

ESPING-ANDERSEN, Gosta, Fundamentos sociales de las economías postindustriales, trad. de Francisco Ramos, Barcelona, Ariel, 2000.

FAjARDo-DolCI, Germán et al., "Acceso efectivo a los servicios de salud: operacionalizando la cobertura universal en salud”, Salud Pública de México, vol. 57, núm. 2, 2015. 
FMI, Perspectivas de la economía mundial, Washington, DC, octubre de 2017, disponible en: https://wmw.imf.org/es/Publications/WEO/Issues/2017/09/19/ world-economic-outlook-october-2017.

FREIJE, Samuel, El empleo informal en América Latina y el Caribe: Causas, consecuencias y recomendaciones de politica, Banco Interamericano de Desarrollo, 2002, disponible en: https://ideas.repec.org/p/idb/brikps/3772.html.

GUAMÁN, Adoración y LORENTE, Raúl, "La afiliación a la seguridad social del trabajo no remunerado del hogar: el modelo de ecuador como ejemplo para un debate necesario", Revista Latinoamericana de Derecho Social, núm. 28, 2019.

HAmilton, Gabriela, "Exclusión de la protección social en salud en Argentina: tres enfoques metodológicos”, Jornadas Gino Germani, Buenos Aires, IIFCS e Instituto de Investigaciones Gino Germani, 2001, disponible en: http:/ / bibliotecavirtual.clacso.org.ar/ar/ libros/argentina/germani/ hamilton.rtf.

INEGI, “Estadísticas históricas de México 2014”, Instituto Nacional de Estadística y Geografía. México, INEGI, 2015, disponible en: https:// wmw. inegi.org. $m x /$ app / biblioteca / ficha. btml? upc $=702825058203$.

IPCC, "Climate Change 2014: Impacts, Adaptation, and Vulnerability. Part B: Regional Aspects. Contribution of Working Group II to the Fifth Assessment Report of the Intergovernmental Panel on Climate Change", en BArros, V. R. et al. (coords.), Cambridge University Press, Cambridge, Reino Unido, Nueva York, Estados Unidos, 2014, disponible en: https:/ / www.cambridge.org/core/services/aop-cambridge-core/content/view/E816A2701 AD893B49639DDDA5E44F72E/9781107415386c1_p1133-1198_CBO. pdf/regional_context.pdf.

JørGensen, Steen y Holzmann, Robert, "Manejo social del riesgo : un nuevo marco conceptual para la protección social y más allá”, Revista Facultad Nacional de Salud Pública, vol. 21, núm. 1, 2003.

KIRSCH, Henry, "La participación de la mujer en los mercados de trabajo en Latinoamérica", Notas de Población, Naciones Unidas, Comisión Económica para América Latina y el Caribe (CEPAL), 1975.

KUZNETS, Simon, "Economic Growth and Income Inequality", Economic Growth and Structure, Nueva York, 1965.

LAURELL, Cristina, Impacto del seguro popular en el sistema de salud mexicano, Buenos Aires, Clacso, 2013, disponible en: http://paginas.facmed.unam.mx/deptos/sp/wp-content/uploads/2015/11/ImpactodelSeguroPopular.pdf. 
Molyneux, Maxine, "Más allá del debate sobre el trabajo doméstico", en RODRíGuEZ, Dinah y COOPER, Jennifer (comps.), El debate sobre el trabajo doméstico, Ciudad de México: Instituto de Investigaciones Económicas, UNAM, 2005.

OfFE, Claus, Contradicciones en el Estado de Bienestar, trad. de Antonio Escohotado, Madrid, Alianza Editorial, 1994, vol. 647.

Organización Internacional del Trabajo, "Protección Social de la Salud: Una estrategia de la OIT para el acceso universal a la asistencia médica. Documento de consulta", Ginebra, Organización Internacional del Trabajo, agosto de 2007, disponible en: https:// wmw.ilo.org/public/spanish/protection/ secsoc/downloads/ healthpolicy_sp.pdf.

Organización Internacional del Trabajo, "Las mujeres en el trabajo: Tendencias de 2016", Ginebra, Organización Internacional del Trabajo, 2016, disponible en: https:// www.ilo.org/wcmsp5/groups/public/---dgreports/---dcomm/--publ/documents/publication/woms_483214.pdf.

OXFAM, "Premiar el trabajo, no la riqueza", Gran Bretaña, Oxfam Internacional, Cowley, enero de 2018, disponible en: https:// www-cdn.oxfam.org/ s3fs-public/file_attachments/bp-reward-work-not-wealth-220118-es.pdf.

OszLAK, Oscar, "Formación histórica del Estado en América Latina: elementos teórico-metodológicos para su estudio", en OSLAK, Oscar (ed.), Lecturas sobre el Estado y las políticas públicas: Retomando el debate de ayer para fortalecer el actual, San José, Costa Rica, EDUCA, 2007.

PIKETTY, Thomas, El Capital en el Siglo XXI, trad. de Eliane Cazenave-Tapie Isoard, México, Fondo de Cultura Económica, 2014.

PIZARRO, Roberto, "La vulnerabilidad social y sus desafíos: una mirada desde América Latina", Estudios estadísticos y prospectivos, Santiago de Chile, CEPAL, 2001, disponible en: https:/ / repositorio.cepal.org/ bitstream/ handle/11 362/4762/S0102116_es.pdf? sequence $=1$ Gis Allowed $=y$.

PREBISCH, Raúl, "Raul Prebisch: un aporte al estudio de su pensamiento", Comercio Exterior, vol. 37, núm. 5, mayo de 1987.

PNUD, “Informe sobre Desarrollo Humano México 2016”, 2017, disponible en: https:// wmw.undp.org/content/dam/mexico/docs/Publicaciones/Publicacio nesReduccionPobreza/InformesDesarrolloHumano/idhmovilidadsocial2016/ PNUD\%20IDH2016.pdf.

RAMíREZ, Berenice et al., "Las raíces de la desigualdad y de la exclusión previsional en México: propuesta para su rediseño", en RoDRíGUEZ, Ignacio y 
Vommaro, Pablo (coords.), Desigualdades, exclusión y crisis de sustentabilidad en los sistemas previsionales de América Latina y el Caribe, Buenos Aires, CLACSO, 2018.

RaWls, John, Teoría de la justicia, trad. de María Dolores González, México, Fondo de Cultura Económica, 2015, 1995.

RENDÓN GAN, Teresa, Trabajo de hombres y trabajo de mujeres en el México del siglo XX, México, CRIM-PUEG-UNAM, 2003.

RODRíGueZ, Corina, "La cuestión del cuidado: ¿El eslabón perdido del análisis económico?”, Revista CEPAL, núm. 106, 2012.

SALGADO-De SNYDER, Nelly y WONG, Rebeca, "Género y pobreza: Determinantes de la salud en la vejez”, Salud Publica de Mexico, vol. 49, supl. 4, enero de 2007.

SÁNCHEZ, Armando et al., "Determinantes de las horas de trabajo de las mujeres en México: un enfoque de pseudopanel (2005-2010)", Revista de la CEPAL, núm. 120, diciembre de 2016.

SECCOMBE, Wally, "El trabajo del ama de casa en el capitalismo", en RoDRíGUEZ, Dinah y COOPER, Jennifer (comps.), El debate sobre el trabajo doméstico, México, UNAM, Instituto de Investigaciones Económicas, 2005.

Sojo, Ana, "Protección Social en América Latina. La desigualdad en el banquillo", Santiago, CEPAL, 2017, disponible en: https:/ / repositorio.cepal.org/ bitstream/handle/11362/41105/6/S1600819_es.pdf.

Bases de datos

Encuesta Nacional de Empleo y Seguridad Social ENESS, tercer trimestre de 2017, Instituto Nacional de Estadística y Geografía, INEGI, disponible en: http:/ / www.beta.inegi.org.mx/programas/eness/2017/default.html.

Encuesta Nacional de Ocupación y Empleo ENOE, tercer trimestre de 2017, Instituto Nacional de Estadística y Geografía, INEGI, disponible en: https:// wmw.inegi.org.mx/programas/enoe/15ymas/. 